\title{
Le concept de théâtre musical en Italie.
}

\author{
De la tragédie de l'écoute de Luigi Nono \\ à l'action invisible de Salvatore Sciarrino
}

\section{Francesca Guerrasio}

Résumé : La notion de «théâtre musical» dans la seconde moitié du $x x^{e}$ siècle, attire l'attention de nombreux compositeurs en Europe. En Italie, l'intérêt pour le théâtre musical se manifeste au cœur de la production et de la réflexion musicales d'avant-garde dans les années soixante. Des compositeurs tels Luigi Nono, Luciano Berio, Salvatore Sciarrino (pour n'en citer que trois) possèdent la volonté de reconstruire le monde musical autour d'une nouvelle approche créative indifférente au scandale, qui mette en lumière la richesse qu'une telle expérience peut apporter, et la volonté communicative qui en résulte. L'absence d'interventions communicatives, des esthétiques arides et des exécutions monotones sont le résultat d'une approche pseudo-scientifique de la musique fondée sur des analyses stériles et sur des calculs mathématiques. Il est nécessaire, pour ces compositeurs, de «communiquer» à l'aide d'un discours qui doit être porteur d'un sens, d'une familiarité, d'une connaissance, d'un amour: d'un rapport affectif et émotionnel touchant à tous les protagonistes/récepteurs d'un tel langage. L'exploration du coté inexploré de l'expérience musicale est à la base d'un renouvellement essentiel de formes de théâtre considérées comme traditionnelles et révolues (comme l'opéra lyrique).

Resumo : A noção de «teatro musical » durante a segunda metade do século XX instiga diversos compositores na Europa. Na Itália, o interesse pelo gênero se manifestou no âmbito da produção e da reflexão musical de vanguarda nos anos sessenta. Compositores como Luigi Nono, Luciano Berio, Salvatore Sciarrino (para citar apenas três) pretendiam reconstruir o mundo musical em torno de uma abordagem criativa, indiferente ao escândalo, valorizando a riquesa desta experiência específica e o impulso comunicativo resultante. A ausência de intervenções comunicativas, as estéticas áridas e as execuções monótonas são o resultado de uma abordagem pseudo-científica da música, baseada em análises estéreis e em cálculos matemáticos. Para estes compositores, é necessário que se comunique com ajuda de um discurso portador de sentido, de familiaridade, 
de conhecimento, de amor: de relações afetivas e emocionais que atinjam à todos os protagonistas/receptores de tal linguagem. A exploração do lado ainda desconhecido da experiência musical é o fudamento de uma renovação essencial de formas teatrais consideradas tradicionais e ultrapassadas (como a ópera lírica).

Dans les années 1950 un nouveau concept de «théâtre musical », basé sur un type différent de fusion d'éléments dramatiques et musicaux, semble annoncer un échange renouvelé entre diverses disciplines artistiques. Chaque expérience qui se démarque des structures narratives traditionnelles est cataloguée comme appartenant au théâtre musical. Dès lors, de nouvelles esthétiques comme le théâtre sonore (Pierre Henry), l'action musicale (Luciano Berio), le théâtre du corps (Brian Ferneyhough), la tragédie de l'écoute et l'action scénique (Luigi Nono), l'action invisible (Salvatore Sciarrino) et d'autres formes d'œuvre ouverte (théorisée par Umberto Eco), de théâtre total, promenades sonores, etc., sont rapportées au genre du «théâtre musical», qui se caractérise par "la multiplicité des signes et des événements à produire simultanément»(Bosseur, 1996, p. 172). La participation active des différents protagonistes de la dramaturgie, de l'instrumentiste au chanteur, tout en passant par l'acteur, le compositeur et le spectateur, donne vie à une démarche collective où chaque participant est "complice» de ses partenaires et partie intégrante d'un projet musical, voire d'un événement qui sous-tend l'approfondissement de «la musicalité du temps dramatique » et la «théâtralité du jeu musical »(Bosseur, 1996, p.171).

En Italie, l'intérêt pour le théâtre musical se manifeste au cœur de la production et de la réflexion musicales d'avant-garde dans les années soixante. Des compositeurs tels Bruno Maderna, Luigi Nono, Luciano Berio, Franco Evangelisti, Sylvano Bussotti, Giacomo Manzoni possèdent la volonté de reconstruire le monde musical autour d'une nouvelle 
approche créative ${ }^{1}$ indifférente au scandale, qui mette en lumière la richesse qu'une telle expérience peut apporter, et la volonté communicative qui en résulte. "La nécessité première », écrit Nono, « est : communiquer» (Nono, 1985, p. 67).

La double exigence de l'évolution musicale «postsérielle » et de l'introduction du gestuel dans une œuvre ouverte devient l'élément déterminant d'une (r)évolution du théâtre musical. Ce dernier étant entendu aussi comme spectacle audiovisuel à l'écart du « théâtre musical traditionnel », autrement dit de l'opéra. Malgré la présence de différences évidentes entre les expériences de ces artistes engagés, il apparaît nettement que tous se penchent sur la volonté de dépasser la «difficulté d'une narration linéaire et continue d'histoires liées à la psychologie des personnages»(Petazzi, 1995, p. 11). Tous cherchent à élaborer une dramaturgie éloignée des schémas traditionnels, à créer une œuvre d'art qui se constitue en tant que signification et parole d'une «conscience authentique» (Adorno, 1982, p. 255). Cette nouvelle forme d'expression éloignée de l'opéra aurait pour but de faire apparaître, dans la formation de sa totalité, dans son idéal de complète structuration, une société plus digne des hommes et l'image d'une humanité transformée (Adorno, 1982, p. 333). D'où l'importance d'une «contagion» esthétique (dans le sens étymologique du terme) comme modification émotionnelle de la sensibilité du destinataire affecté par la musique.

Dans les faits, le concept d'opéra lyrique «vieux, révolu et dépassé », voire le concept d'une dramaturgie assurée par la lecture musicale d'un livret, «donc d'un texte porteur de situations dramatiques » (Aperghis, apud Gindt, 1990, p. 61) n'est pas forcément

\footnotetext{
1 Allezhop de Berio et Calvino fut représenté à la Biennale de Venise en 1959 ; Sentenza de Manzoni composée en 1959 fut mise en scène en 1960; Intolleranza de Nono fut au centre d'un célèbre scandale à Venise en 1961 ; Passaggio de Berio (sur un texte de Sanguineti) des années 1961-62 fut accueilli sans succès à la Piccola Scala en 1963 ; Hyperion de Maderna prit forme en 1964 mais le compositeur continuera à le travailler jusqu'en 1969 comme une sorte de work in progress; Atomtod de Manzoni sera présenté à la Piccola Scala en 1965.
} 
abandonné mais plutôt modifié. Les éléments caractéristiques de l'opéra: scénographie, mise en scène, division en actes, etc., restent souvent à la base des nouvelles expérimentations, mais à ceux-ci s'ajoutent des signaux multiples envoyés à l'auditeur-spectateur. On assiste parfois à l'éclatement du récit et à la dissociation des composantes du spectacle musical qui retrouve une autonomie propre dans le cadre d'un canevas autrement structuré. La partition régit tous les événements principaux et secondaires de la dramaturgie, du texte porteur d'un sens au geste sonore, corporel et vocal, qui donne naissance à " une certaine dramaturgie de l'indicible » (Aperghis, apud Gindt, 1990, p.62). Le théâtre musical semble donc coïncider avec «l'envahissement du temple théâtral par le pouvoir abstrait de l'organisation musicale » (Aperghis, apud Gindt, 1990, p. 62).

On remarque une volonté d'élaborer une situation ouverte à plusieurs horizons, dépassant la dichotomie théâtre-musique pour créer une nouvelle forme de spectacle musical avant tout fondé sur l'art "dramatique-sonore », voire sur une dramaturgie en tant que principe organisationnel, et de la musique, et du texte. Musique et théâtre s'associent pour solliciter la participation du spectateur-auditeur et de l'instrumentiste ${ }^{2}$-chanteur-acteur vers une écoute qui n'est plus monodirectionnelle et une situation qui n'est plus mono-significative.

Cette nouvelle pensée veut refonder l'événement théâtral en musique au moyen de divers procédés :

- $\quad$ les sujets changent ;

- $\quad$ la « monotonie » du procédé narratif linéaire est brisée ;

\footnotetext{
2 La participation théâtrale de l'instrumentiste n'est pas à sous-évaluer. Son rôle n'est pas moins important, dans certains cas, que celui du protagoniste de l'opéra. Le musicien doit pouvoir créer sa «vie scénique » de façon intense et instaurer avec son propre instrument un lien fort. Ce dernier étant compris comme son prolongement corporel. Ses mouvements, une fois pris en compte dans la composition musicale, deviennent la «marque» essentielle des expérimentations de Sciarrino, qui bouleverse de façon étonnante le caractère statique et anonyme de l'instrumentiste dans l'opéra traditionnel.
} 
- $\quad$ la parole en musique perd sa fonction d'expression ou de mise en valeur des sentiments ;

- l'organisation spatiale se modifie et de nouveaux lieux de représentation apparaissent.

Cette période historique accouche d'une vision plurielle de la dramaturgie musicale, d'un point de vue esthétique, langagier, matériel et spatial.

Le ballet, la pantomime et le spectacle de marionnettes ${ }^{3}$ sont, en Italie, les premiers résultats de cet esprit de renouvellement. Ils sont bientôt suivis de la radiophonie où s'installe progressivement la dramaturgie musicale. La perspective d'échapper à l'institution du théâtre lyrique explique le développement considérable de la production musicale radiophonique à partir des années 1950. Il s'agit d'opéras radiophoniques: des «radio-drames» qui instaurent l'une des premières approches du drame en musique. À ce propos, il est significatif qu'un compositeur comme Bruno Maderna considère Don Perlimplin4 (1961), opéra radiophonique, comme son premier projet de dramaturgie musicale. Notons également que l'un des premiers opéras de Salvatore Sciarrino, Lohengrin, azione invisibile, est une commande de la RAl, Radio Televisione Italiana en 1980.

\footnotetext{
3 Le spectacle de marionnettes est une des activités théâtrales italiennes que l'on ne doit pas sous-estimer. On trouve ces spectacles du nord au sud de I'Italie. Je ne fais pas seulement référence au renouvellement de spectacles traditionnels tels que l'Opera dei pupi en Sicile ou le théâtre Vecchio San Carlino à Naples, mais aussi à l'Opera dei Burattini que Maria Signorelli crée à Rome en 1947 avec la collaboration de metteurs en scène tels que Lina Wertmuller et Nello Risi, de scénographes comme Franco Laurenti ou Enrico Prampolini, et de compositeurs comme Ennio Morricone ou Roman Vlad. La marionnette n'est pas un spectacle populaire ou enfantin mais une forme théâtrale "prometteuse et proche du monde de la musique ». FERRARI, Giordano, Les débuts du théâtre musical d'avant-garde en Italie. Berio, Evangelisti, Maderna, Paris, I'Harmattan, 2000, p. 19.

4 D'après les sources de la RAI, Radio Televisione Italiana, Don Perlimplin aurait été présenté au Prix Italia en 1961 mais d'autres sources indiquent l'achèvement de l'opéra en 1962.
} 
La radio permet la naissance d'une nouvelle approche de la vocalité : grâce à l'utilisation du microphone, la parole prend une importance fondamentale dans la diction d'un texte. Les accents, les soupirs, les variations de ton ou encore la vitesse de lecture ont une importance capitale à la radio. Ce véritable univers sonore offert par la voix est particulièrement exploité par Luciano Berio ${ }^{5}$ avec la complicité de Cathy Berberian. Leur collaboration donne vie à Thema Omaggio à Joyce (1958), une lecture inédite d'un extrait du chapitre XI de l'Ulysse de Joyce. Cette expérimentation témoigne de l'intérêt pour la recherche et développe une nouvelle approche de l'écriture, qui s'éloigne de la théâtralité, pour aller à l'encontre de la dramaturgie du récit et transmettre des idées à la fois politiques, sociales et existentielles.

Parmi les réponses significatives apportées aux problèmes du théâtre musical ${ }^{6}$, se distingue Intolleranza 1960, azione scenica (action scénique ${ }^{7}$ ) de Luigi Nono. La présentation de ce livret tranche par rapport à la tradition: constitué à partir d'une idée d'Angelo Maria Ripellino (matériau de départ), il se compose d'un ensemble de textes différents 8 . Le récit se développe à travers des séquences non linéaires, des situations et des moments, qui ne racontent pas tant une histoire que la

\footnotetext{
5 Berio a surtout orienté ses recherches sur le phénomène vocal. Dans l'œuvre Thema Omaggio a Joyce (1958), composé au Studio de Phonologie de Milan, « il a réussi à créer un passage continu entre l'électronique et la voix humaine ». $C f$. Bosseur, Jean-Yves, 1973, p. 44.

6 Pour une étude plus approfondie des œuvres de théâtre musical chez les compositeurs italiens à partir de 1949 jusqu'à 1970, voir le schéma de FERRARI, Giordano, Les débuts du théâtre musical d'avant-garde en Italie. Berio, Evangelisti, Maderna. Paris : L'Harmattan, 2000, p. 38-42.

7 Selon Jürg Stenzl, dans les archives des éditions Schott, à Mayence, une lettre de Nuria Nono interdit expressément d'utiliser le mot «opéra » dans les éditions de la partition et du livret de Intolleranza 1960. Cela témoigne la volonté de s'éloigner de ce genre théâtral. Cf. STENZL, Jürg, "La dramaturgie musicale de Luigi Nono », Contrechamps n.4, 1985, p. 68-72.

8 L'idée du départ est Vivere è stare svegli de Ripellino auquel s'ajoutent : cinq strophes du poème La liberté d'Eluard ; Unser Marsch de Maïakoski ; An die Nachgeboren de Brecht ; des slogans historiques tels que : Morte al fascismo!, Libertà ai popoli !... ; des extraits d'interrogatoires nazis de Julius Fucik et de la police française en Algérie ; des discours de Henri Alleg ; un extrait de la préface de Sartre au livre d'Alleg, La question. Pour une reconstruction complète voir STENZL, Jürg, op. cit., p. 69-79.
} 
naissance d'une conscience actuelle, active et sociale, comme pourra le dire Nono $^{9}$. Cela se produira souvent dans le théâtre musical de cette période. Les principales caractéristiques formelles à proscrire sont décrites par le compositeur et exposées pour la première fois en 1962, lors d'une conférence donnée sur l'île San Giorgio, à Venise ${ }^{10}$.

On peut les résumer ainsi :

1. séparation entre public et scène comme dans un rituel antique ;

2. rapport simpliste entre la dimension visuelle et sonore de l'opéra selon lequel «je vois ce que j'écoute, et j'entends ce que je vois »;

3. fonction d'illustration scénico-visuelle de la situation chantée ;

4. rapport univoque entre chant et orchestre ;

5. perspective focalisée sur l'unicité des plans visuels et sonores: «on bloque [ainsi] toute possibilité d'utiliser le rapport espace-temps» (Nono, 1985, p. 59)

Pour Nono (tout comme pour Sciarrino dix ans plus tard) il est fondamental d'échapper au rituel 11 ainsi décliné pour pouvoir bâtir un nouveau théâtre musical.

L'opéra, Prometeo, tragedia dell'ascolto, que l'on peut considérer comme l'une des œuvres-phares du renouvellement italien des années 1980, met en évidence la problématique du rapport musique/image et

9 Comme l'écrit Jürg Stenzl, «ce réseau textuel ne raconte pas en premier lieu une histoire, mais rend intelligible, sous la forme d'une focalisation de moments isolés, la naissance d'une conscience actuelle, active et sociale ». STENZL, Jürg, op. cit., p. 70 . Traduction de l'auteur.

10 La conférence a eu lieu le 27 septembre 1962 ; ele été traduite et publiée dans la revue Contrechamp (NONO, 1985), réédité récemment (FENEYROU, L. (dir.), Écrits. Paris: Christian Bourgois, 1993, p. 213-233.

11 Comme l'explique Nono, il faut tenir compte du fait que la distance entre public et scène trouve son origine non seulement dans la pratique liturgique mais aussi dans les premières représentations des Mystères et des Sacre rappresentazioni du Moyen Age. On dressait sur les places des scènes fixes, autour desquelles le public se rassemblait. De cette façon on annulait toute sorte de rapport social entre action scénique et public. NONO, Luigi, "Possibilité et nécessité d'un nouveau théâtre musical », Contrechamps, Lausanne, L'Âge d'Homme, 1985, n 4, p. 59. 
revalorise le silence à l'intérieur d'un processus sonore narratif. Constitutif et enrichissant pour le compositeur, ce processus l'est également pour l'interprète et l'auditeur-spectateur.

À la conception «statico-théologique ${ }^{12}$ », Nono substitue une vision dynamique de relations variables:

- $\quad$ plus grande richesse des éléments et des dimensions dans la composition théâtrale ;

- $\quad$ plus grande participation du public ;

- $\quad$ possibilité d'avoir des sources visuelles et sonores disséminées dans toute la salle. Ce qui se traduit par la libération du rapport spatio-temporel.

L'architecture/espace sonore est conçue comme un instrument musical, dans lequel le son peut s'épanouir et l'écoute se libérer de l'esclavage de la vision ${ }^{13}$. Il ne s'agit plus de mettre au point une méthode de composition rationnelle mais de faire un exercice (d'écriture et d'écoute), qui implique l'ouverture à l'autre et revalorise la subjectivité.

Nono (comme l'avaient fait avant lui Edgard Varèse, lannis Xenakis, Karlheinz Stockhausen) est sans doute le compositeur du $x x^{e}$ siècle qui a introduit en Italie, de la façon la plus conséquente, la spatialisation en tant que principe fondamental, structurant toutes les dimensions du langage musical. Sa tragédie de l'écoute attribue, en outre, une importance considérable au silence : possibilité d'ouverture à

\footnotetext{
12 Selon Nono l'opéra traditionnel européen était basé sur un foyer unique ; une source sonore unique ; un rapport liturgique entre public et scène. Ibidem.

13 L'originalité de Nono consiste à ne plus pouvoir considérer des œuvres "autres que politiques », et à entretenir des nouvelles relations entre le compositeur et les masses populaires. Ce qui l'amène naturellement à rompre avec les circuits de distribution habituels du monde bourgeois (les festivals par exemple) et à chercher d'autres lieux d'écoute : sortie d'usine, halls, lieux divers. Cf. JAMEUX, Dominique, Luigi Nono, Musique de notre temps. Paris : Casterman, 1973, recueil 1, p. 173-175. Ouvrage collectif en 46 fiches sous la direction de Georges Kadar.
} 
l'autre, phénoménologie d'écoute du «moi étranger» (une conception bien en phase avec l'aspiration de Sciarrino à une musique écologique).

Dans les mêmes années, Un re in ascolto de Berio devient le paradigme d'une nouvelle pratique de la mise en scène et de ses conséquences possibles. Il s'agit d'une œuvre construite sur un effet de théâtre dans le théâtre, solution de métathéâtre déjà expérimentée par Luigi Pirandello dans le domaine de la littérature italienne. "J'ai rêvé d'un théâtre » affirme Prospero ${ }^{14}$, à la recherche de quelque chose qui est «dit entre les sons » et qui amène à l'éclatement du statut du personnage comme conséquence de la multiplicité des niveaux possibles d'appréhension de la réalité. Un théâtre réinventé, une alternative au théâtre aristotélicien, basé sur la trama : qui est là, même si on ne le voit pas parce que la musique se déroule toujours sur une scène intérieure, évoquant une façon imaginaire d'être physiquement. Un théâtre qui s'identifie de plus en plus avec un théâtre de l'écoute où la notion de temps directionnel se trouve suspendue, «la téléologie devient ataraxie »(Bossis, 2002, p. 256). C'est la puissance d'histrion du geste, de suggestion du mime et de la danse qui se conclut avec une sorte de rituel d'adieu, «une initiation à la mort de l'opéra » avec laquelle Berio semble démontrer l'impossibilité de la scène opératique.

À cette époque, Nono achève son Prometeo, qui est une tragédie de l'écoute, Berio son $R e$ in ascolto : la problématique de l'écoute aboutit à un éclatement du statut du personnage et à celui de la représentation ; Sciarrino pose les fondements poétiques et musicaux d'une dramaturgie de l'écoute à travers la métaphore de I'action invisible ${ }^{15}$. Lohengrin, I'un de ses premiers opéras, est une véritable tragédie, qui confirme la prédilection du compositeur pour un théâtre musical de l'intériorité et une «cosmogonie vocale et sonore» (Vinay, 2003, p. 813). Elsa,

\footnotetext{
14 Texte original : Ho sognato un teatro. BERIO, Luciano, Un re in ascolto, azione musicale in due parti, Milan, Universal Edition, 1983.

15 Pour une explication détaillée du concept d'action invisible chez Sciarrino, je renvoie au deuxième chapitre de cette première partie.
} 
protagoniste/actrice, rassemble en elle-même toutes les fonctions de représentation: elle est décor dans la représentation virtuelle de son histoire infinie. Elle est musique, dans les bruits, les palpitations, les aboiements et les silences qu'elle produit. Elle est le reflet de sa conscience troublée et d'une condition existentielle dans laquelle l'attente ne peut pas trouver de réponse. L'oscillation incessante entre imagination et réalité, entre images phonétiques, scéniques et vocales, trouve dans la musique l'élément de cohérence de toute la partition. La clé des événements n'étant plus l'image mais l'événement sonore.

\section{À la recherche d'une nouvelle vocalité}

L'envie de renouveau ne se limite pas à la recherche d'un espace pour la musique ou d'une simple esthétique apparente, mais touche aux éléments intrinsèques du fait musical. $\mathrm{Si}$, de tout temps, la voix a retenu l'attention des compositeurs et passionné le public, il faut attendre le xxe siècle pour que la notion de vocalité soit véritablement élargie, pour être considérée alors comme "la qualité vocale d'un événement sonore » (Bossis, 2005, p.8).

La plupart des options en matière vocale sont plus ou moins directement issues de la conception wagnérienne. C'est ainsi qu'en 1909 Arnold Schoenberg crée un monodrame, Erwartung, dont le thème est aux frontières de l'inconscient. L'unique personnage se trouve dans un tel état d'exaltation que sa voix suit une ligne musicale constamment brisée (ce qui arrive de façon différente mais analogue chez les personnages féminins des opéras de Sciarrino). Cependant, on est encore dans le domaine d'un chant (au sens traditionnel du mot). La véritable (r)évolution vocale date de 1911, année où le Pierrot lunaire de Schoenberg ouvre un nouvel espace dans l'utilisation de l'organe phonatoire. Le parlé se pare d'inflexions déjà modulées et le chanté se rapproche beaucoup du soupir et des susurrements. 
Le Bel Canto ${ }^{16}$, avec ses prouesses vocales incontournables, a marqué une certaine époque ; les grandes voix ne s'adaptent plus à ce genre de déclamation/chant où un son de hauteur précise se mêle à une phrase en parlando. Place maintenant à la technique du Sprechgesang, basée sur les élans et les retombées, sur différentes techniques d'émission : avec la gorge serrée, tendue, la bouche ouverte comme un soupir d'accablement, les accents marqués par de forts coups de diaphragme. Place au Stimmung ${ }^{17}$ où une vaste gamme de modes de jeu traduit différents états intérieurs, dont le plus net est le désenchantement.

À partir de là, de nombreuses expérimentations sont menées afin d'explorer de plus en plus les matériaux linguistiques, ainsi que les possibilités de l'appareil phonatoire humain. À ce propos, il nous semble important de rappeler l'intérêt, et même la passion, que Sylvano Bussotti, fidèle à ses origines transalpines, a manifestés pour la voix. Rara Requiem (1969) exploite les matériaux verbaux avec des intentions strictement vocales : les consonnes y jouent un rôle primordial. Qu'elles soient roulées (double « $r »$ ) ou sifflées, elles sont projetées de la cavité buccale avec une force certaine, qui contraste avec le chuchotement de certains mots ou l'esquisse des rires. Ce qui dénote un désir d'expérimentation et une approche intellectuelle du traitement vocal. Sa ligne musicale est caractérisée par de grands intervalles, qui obligent la voix à basculer d'un registre à l'autre. Mais son style est encore proche du chant traditionnel : la voix est soit très vibrante, soit bien droite, et

\footnotetext{
16 Le bel canto donne une bonne occasion de s'interroger sur l'aspect technique de l'art du chant. La vocalise apparaît souvent à la fois comme l'exercice incontournable et comme la prouesse où se mesure la virtuosité du "chantre ». TROTTMANN, Christian, La voix enchantée. Essai philosophique sur le chant, Éditions Universitaires de Dijon, 1998, p. 80.

17 Pour une plus large réflexion et un approfondissement des diverses techniques ou «modalités constitutives» de la voix dont la Stimmung représenterait "un état d'harmonie et de résonance entre une voix et les affects de l'âme » voir COHEN-LEVINAS, Danielle, "La voix inactuelle : trois intraduisibles de son et de sens - Stimme, Sprechgesang, Stimmung», La voix au-delà du chant, Paris, Vrin, 2006, p. 224-233. Nouvelle édiction recomposée et augmentée.
} 
les modes d'attaque, souvent aux antipodes les uns des autres, trahissent une certaine incongruité dans le contexte général de l'œuvre.

$\mathrm{Au}$ sommet de la production contemporaine italienne figure l'œuvre vocal de Luciano Berio, compositeur italien, passionné par la voix, qui a grandement contribué à l'évolution dans le domaine des possibilités vocales. Son travail, à partir des recherches de Cathy Berberian et en étroite collaboration avec elle, parvient à secouer la parole jusqu'à ses racines phonétiques pour aboutir à une négation du langage conventionnel, malgré la présence d'un texte initial. Sa Sequenza II/8 (1965) est sans doute l'œuvre majeure de l'histoire du renouvellement du «chant» contemporain. Le trait dominant de sa partition (dont le texte est en anglais) est le jeu de contrastes entre les sons tenus, et le nombre de gestes vocaux, allant du rire au parlé dans une mobilité incessante d'états d'âme : urgent, apprehensive, extremely intense, incresingly desperate, anxious, very excited and frantic, ecstatic. Le «Leitmotiv» de sa composition est l'intention de briser la cohérence d'un texte intelligible pour dévoiler une nouvelle approche dramatique et dramaturgique de la conscience individuelle. Ce jeu psychologique ne renie pas la tradition: parmi ce foisonnement sonore, les trilles, les trémolos, les ornements, les glissandi s'insèrent congrûment dans la partition cohabitant avec les bruits vocaux. Pour l'exécution, Berio ne recherche pas une chanteuse, mais plutôt une actrice, qui sache approfondir la richesse d'une telle partition psychologique, qui sache en interpréter l'imagination créatrice, relever les mots les plus frappants et moduler les mille nuances vocales. Et pour satisfaire à ce rôle, qui pouvait être mieux placé que sa complice, Cathy Berberian?

Evidemment, dans le panorama des expérimentations, bien d'autres compositeurs ont ouvert de nouvelles pistes de recherche: Stockhausen par son important travail sur le souffle, Schnittke avec les

18 BERIO, Luciano, Sequenza III, pour voix de femme, composée sur un texte en anglais de Markus Kutter, Londres, Universal Edition, 1968. Interprétée par Cathy Berberian; durée environ huit minutes. 
innombrables onomatopées de son Ursonate. Cependant, tant au niveau intentionnel qu'au niveau de la composition, les compositeurs italiens comme Berio, Bussotti, Nono et Sciarrino ont joué un rôle fondamental dans l'évolution de la vocalité en Italie.

Ce dernier pousse son travail à la limite de l'exploration vocale, considérant la voix comme émanation du corps tout entier, dont il faut avoir une maîtrise exceptionnelle si l'on aspire à moduler toutes ses intentions sonores. Lieu d'intériorité, de fidélité des différents mouvements de l'âme, elle se traduit dans un mode d'expression de la souffrance devant un éternel devenir: la voix d'un «espace infini » (Barthes, 1982, p. 240). À partir de Lohengrin, Sciarrino charge l'événement vocal d'une grande portée psychologique, à travers l'évocation de la nature et des mouvements introspectifs.

La vibration de l'air émis par le corps humain prolonge l'individu à l'extérieur de lui-même. Elle lui permet d'envoyer en délégation dans l'espace la trace immatérielle mais physique de ses émotions et de ses pensées, pour la simple «traversée d'un lieu » comme dans l'appel, pour investir son territoire social structuré dans la langue par l'échange de paroles ou [...] l'ornement modulé du chant ${ }^{19}$.

Libérée de sa connotation «négative », de la nécessité de dire, la voix s'aventure dans les territoires inexplorés de la conscience humaine, caractérisés par des bruits, des cris animalesques et une infinitude de sons presque inaudibles. Citons à titre d'exemple les premiers gestes «vocaux» d'Elsa, le protagoniste de Lohengrin, produits dans des halètements qui rappellent des aboiements lointains renvoyant à l'image sonore d'un paysage nocturne champêtre. Il ne s'agit pas simplement de

19 MÂCHE, François-Bernard, POCHÉ, Christian, La voix maintenant et ailleurs, Paris, coédition de la BPI et du Centre régional de la voix de la Fondation Royaumont, s.p. Exposition de la Bibliothèque publique d'information du Centre Georges Pompidou, avec la participation du Centre régional de la voix de la Fondation Royaumont. 
l'imitation d'un espace mais plutôt de son évocation à travers des sons aptes à susciter de pures illusions : aptes à reproduire à travers la voix le « monstrueux paysage de l'âme ${ }^{20}$ ».

Pour la «chanteuse », cela nécessite un tractus vocal totalement dégagé et une grande souplesse des muscles linguaux et bucco-faciaux. La production d'impulsions assez fortes du diaphragme, des petits coups de langue, l'intonation à peine audible de syllabes ou de voyelles ${ }^{21}$, les variations rapides de hauteur (plus aigu, plus grave) et de timbre, le passage d'un son plus accentué à un autre non accentué, des jeux de langue et une respiration très particulière sont les caractéristiques les plus fréquentes du jeu technique demandé par Sciarrino. La perception de ces variations nécessite une forte concentration de la part de l'auditeur, car les caractéristiques phonatoires et acoustiques de l'événement vocal sont parfois masquées par le spectre sonore de l'orchestre ${ }^{22}$. De ce fait, il est difficile de distinguer un micro-son entonné d'un halètement, simple respiration d'un souffle sonorisé.

L'usage de ce dernier, aussi abondant que diversifié, se révèle un trait fondamental de la poétique vocale de Sciarrino. Des soupirs forcés alternant avec de longs soupirs étirés, le parlando alternant avec du souffle, ou encore de véritables exhalations alternant avec des inspirations sonorisées, tous ces éléments traduiront les sentiments très communs de la surprise, de la peur, de l'angoisse, de l'attente. Le souffle est donc chargé d'un rôle très important: celui d'évoquer, au même degré que les sons musicaux, toute une série d'événements objectifs

20 SCIARRINO, Salvatore, "Lohengrin, un fanciullo che rifiuta di crescere", Lohengrin, azione invisibile, Milan, Ricordi, 1984, s.p.

${ }_{21} \mathrm{Au}$-dessus de $659 \mathrm{~Hz}$, on ne peut pas réellement distinguer les voyelles $o, u$, $a$, les unes des autres car nous sommes dans la zone d'inintelligibilité absolue.

22 Les fréquences de la voix varient autour de $650 \mathrm{~Hz}$. Celles du violoncelle autour de $1000 \mathrm{~Hz}$, et celles du violon autour de $2660 \mathrm{~Hz}$. Selon la théorie de Leipp et Castellengo de 1969, "Superposer à une forme acoustique vocale d'autres formes acoustiques équivaut nécessairement à un masquage réciproque plus ou moins important ». SCOTTO DI CARLO, Nicole, "L'intelligibilité de la voix chantée », Médecine des Arts, Revue trimestrielle, Montauban, Alexitère éditions, décembre 1994, n¹0, p. 12. 
(par exemple lors de l'évocation de la nature) et subjectifs (dans le court-circuit de la mémoire des protagonistes des œuvres du compositeur). Défini comme «propulsion d'une certaine quantité d'air à travers le nez ou la bouche ${ }^{23}$ ", il est émanation du corps tout entier, matériau sonore qui a exactement le même statut que le son: "un composé de souffle et de feu ", selon l'interprétation du texte sanscrit Sangita Makaranda24. C'est pourquoi souffles respiratoires, tensions du larynx, roulements de gosier, coups de langue, glissandi sourds, sons aphones, mots chuchotées, illustrent une vision éclatée de la voix, «comme une descente dans les limbes d'une vocalité virtuelle ${ }^{25}$ » où « retrouver le corps, c'est d'abord avouer le souffle 26 ».

Comme l'affirme Sciarrino, « au-delà de certaines limites, il y a le bouleversement des oppositions $27 »$ : le silence ne représente plus le vide ou le rien, mais un espace à remplir avec des sons intimes provenant de la mémoire de ses personnages, de leur inconscient et, souvent, de leur conscience troublée. Son et souffle ne sont en définitive que deux éléments d'un unicuum indissociable. Quant aux bruits, pris pour leur valeur réaliste (les aboiements des chiens à la campagne dans Lohengrin, le vent dans La perfezione di uno spirito sottile ${ }^{28}$, les gouttes d'eau dans Infinito nero 29 , les mots chuchotés partout dans les œuvres du compositeur), ils ouvrent sur un champ poétique immense, sur un art qui

23 MÂCHE, François-Bernard, POCHÉ, Christian, La voix maintenant et ailleurs, Paris, coédition de la BPI et du Centre régional de la voix de la Fondation Royaumont, s.p. Selon F.-B. Mâche, le souffle est la propulsion d'une certaine quantité d'air à travers le nez ou la bouche. On produit ainsi un bruit sourd qui n'est pas un son musical. L'identification d'un «non son» se trouve aux antipodes de la poétique vocale de Sciarrino.

24 MÂCHE, François-Bernard, POCHÉ, Christian, La voix maintenant et ailleurs, Paris, coédition de la BPI et du Centre régional de la voix de la Fondation Royaumont, premier chapitre, s.p.

$25 \mathrm{Ibid}$.

26 Ibid.

27 SCIARRINO, Salvatore « Note sur la partition », Soffio e forma, Milan, Ricordi, 1995, s.p. Traduction personnelle.

28 SCIARRINO, Salvatore, La perfezione di uno spirito sottile, Milan, Ricordi, 1985. Composition pour flûte, voix et percussions aériennes.

29 SCIARRINO, Salvatore, Infinito nero, Milan, Ricordi, 1998. 
s'écarte radicalement de l'écriture vocale traditionnelle. Avec Sciarrino, la voix, libérée de la nécessité de dire, sera l'unique et le dernier refuge de la liberté des personnages. Fragmentée, disloquée, cachée et à peine prononcée, elle perd sa tâche habituelle de porteuse de signification pour naître et mourir dans un son vivant qui la traduit, éventuellement la déroute, dans l'abîme des infinis signifiants. Dans une société décrite par le compositeur comme entièrement soumise aux jugements d'autrui, aux lieux communs et aux fausses pudeurs, elle subsiste singulière, originale, unique, comme un phénomène inaliénable échappant à toute mainmise : «scarification de chacun 30 ».

\section{Le modèle sémiotique dans l'œuvre de Salvatore Sciarrino}

La voix, en tant que matériau sonore, est chargée d'une portée essentielle et par rapport à l'idée de langage et par rapport à l'idée de création artistique, selon Sciarrino; elle est donc déterminante pour la formation d'une chaîne communicative. S'il est vrai que la voix doit être appréhendée comme du son humain articulé, de la phonation en acte, ce qui se révèle intéressant n'est pas la signification de la parole, mais plutôt la mise en œuvre d'un processus «physio-psycho-cognitif31 » complexe qui l'inclut. Autrement dit, ce n'est pas l'apparence de l'expression qui est déterminante mais sa substance ${ }^{32}$. Cela implique la façon manifeste d'écrire cette substance, en un mot le graphisme, qui

\section{Ibid.}

31 MOLINIÉ, Georges, "La voix: les effets de la négativité », Vocabulaire de la voix, Paris, L'Harmattan, 2008, p. 86. Textes réunis et présentés par Barbara Cassin et Danielle Cohen-Levinas.

32 Ibid. Georges Molinié propose une différenciation de la substance de l'expression relevant d'autres langages, d'autres systèmes sémiotiques. "Par exemple, le son humain non articulé ou le son non humain, du volume, de la couleur, du mouvement. II s'agit d'autre chose que de la voix, et pourtant ce sont bien aussi chaque fois d'authentiques constitutions langagières ». Suivant son exemple on précisera donc que "la voix est constituée de la substance de l'expression du langage verbal naturel », où le terme "naturel » implique le son vocal comme le bruit et renvoi au concept d'écologie acoustique (tout « endroit » naturel intéressant du point de vue acoustique) élaboré par Sciarrino. 
décrit, du point de vue de la sémantique, l'aire ratio-conceptuelle de l'événement vocal. Pour parvenir à mélanger son et silence, traduire le geste vocal et susciter de cette façon l'incertitude métaphysique, Sciarrino élabore un système complexe de signes: un ensemble d'éléments linguistiques et musicaux coordonnés par une unité fonctionnelle de prescriptions et de gestes qui présupposent un échange mutuel entre l'interprète et le compositeur 33 .

La fonction expressive de l'événement vocal n'est pas seulement assignée au signe graphique conventionnel mais aussi à l'explication ponctuelle de son «effet psychologique ${ }^{34}$ ». Le matériau vocal découvre ainsi sa spécificité, non plus dans le sens d'une autonomie textuelle, mais dans son interférence avec toute une série d'articulations sémiotiques qui changent le phonème isolé dans un Babel linguistique ; le fragment contracté en une succession de signifiants psychologiques ; le plus petit point sur la portée en un flux continu de souffle et de micro-sons. Respiration/souffle, salive/parole précisément codifiées expriment les diverses manifestations ou acceptions de l'image fondamentale du monde apparent, voire mondain ${ }^{35}$ et de ses renvois

33 Marianne Pousseur, qui est une des interprètes de référence des œuvres de Salvatore Sciarrino et en particulier de Lohengrin, décrit son rapport avec le compositeur comme un moment essentiel pour la compréhension de l'atmosphère auditive à récréer. "Toutes les nuances possibles se retrouvent dans Sciarrino et c'est ça qui rend la chose amusante à travailler et très intéressante à l'écoute. [...] J'ai eu le grand plaisir de travailler avec Salvatore Sciarrino [...] et il explique très bien le type d'atmosphère auditive à laquelle faire référence [...] ». GUERRASIO, Francesca, «Entretien avec Marianne Pousseur», réalisé à Bruxelles le 17 avril 2006, inédit.

34 Voir le schéma $n^{\circ} 2$ : "Système des signes et description gestuelle », p. 29.

35 Comme l'affirme Georges Molinié, «selon la théorie sémiotique de la mondanisation, les langages ont pour unique fonction de médiatiser, par rapport à la subjectivité, l'extériorité massive et informe du monde, pour la traiter par des procédures d'élaboration et de plus ou moins réductrice catégorisation, ce qui aboutit à créer, à construire du mondain. Dans cette optique, on peut admettre que le langage verbal [exprimé par la voix] dit le mondain (et non le monde) : c'est le seul langage à dire le mondain, ce qui détermine à la fois son privilège, sa spécificité, et sa limite. La voix et la musique appréhendables comme éléments d'un système langagier (la voix) et comme production reçue à régime d'art (la musique) relèvent-elles de l'univers des dispositifs, ou des mises en jeu, des procédures de mondanisation [...] ». MOLINIÉ, Georges, "La voix : les effets de la négativité », Vocabulaire de la voix, Paris, L'Harmattan, 2008, p. 82. 
psychologiques. Cet «arsenal» de moyens et de matériaux vocaux utilisés, qui sont à la base de la poétique du temps de la voix, voire de ce que Danielle Cohen-Levinas appelle les «icônes de la voix », révèle l'existence de deux systèmes sémiotiques différents identifiables sur la base des rapports existant entre les nombreux symboles :

1. le système de signes/symboles musicaux à proprement parler ;

2. le système verbal d'indications gestuelles (qui décrit et explique le signe graphique).

Le premier système fait référence au signe de l'écriture musicale (et à sa négation) dont la synthèse révèle la présence non déclarée quoique essentielle et indéniable de l'interrogation du geste; le second système consiste en un ensemble hétérogène strictement référentiel, où à un symbole graphique (conventionnel ou non) correspond une indication verbale voire une " prestation ${ }^{36}$ » de caractère gestuel.

Le résultat est celui d'un résumé formel de l'union du signe et de la parole: d'un métalangage qui convoque inévitablement le corps :

Un lien fonctionnel lie en effet à la voix le geste : comme la voix, il projette le corps dans l'espace de la représentation, et vise à conquérir celui-ci, à le saturer de son mouvement. Le mot prononcé n'existe pas (comme le fait le mot écrit) dans un contexte purement verbal : il participe nécessairement d'un procès général, opérant sur une situation existentielle qu'il altère en quelque façon et dont la totalité engage les corps des participants 37 .

Si l'on accepte que la projection d'un son, qu'il se réfère ou non à un texte, comporte l'affirmation implicite de la présence humaine et de

36 Voir DALMONTE, Rosanna, LORENZINI, Niva, AZZARONI, Loris, FRASNEDI, Fabrizio, /l gesto della forma, Milan, Arcadia Edizioni, 1981.

37 ZUMTHOR, Paul, La lettre et la voix, Paris, Editions du Seuil, coll. "Poétique », 1987, p. 15. 
son effet, il va de soi que cette union indivisible de la voix et du corps est déjà en soi une forme de théâtre où la voix joue "une fonction catalysatrice $38 »$. En se manifestant et en révélant l'évident et/ou l'indicible, elle affirme son privilège, sa spécificité et parfois sa limite. Dans le schéma intitulé : "Système d'indications d'expression » (Fig.1), nous avons résumé les signes graphiques caractéristiques de l'écriture de Sciarrino, leur description gestuelle précisément indiquée dans la partition et l'effet psychologique qui lui est relié. Nous avons pris comme référence trois œuvres du compositeur particulièrement significatives: Lohengrin, Perseo e Andromeda et Infinito nero.

Écrites dans l'espace temporel d'une décennie, ces œuvres ont une importance singulière dans le catalogue de Sciarrino: Lohengrin est, en effet, la première expression de sa recherche d'action invisible; Perseo e Andromeda, sa première œuvre mixte (écrite pour voix et live electronics) et Infinito nero l'accomplissement idéal de cette action invisible exprimée au travers d'une représentation pauvre, voire «nue ». Le système d'indications expressives relatives à ces trois compositions subit une sorte de dessèchement explicatif qui s'accompagne d'une place importante accordée au geste vocal. Cela pourrait s'expliquer par la volonté du compositeur de ne pas alourdir la partition avec des indications dramaturgiques trop précises qui pourraient briser l'architecture de l'œuvre, donc la fonctionnalité des espaces, et de laisser à l'interprète une marge « d'improvisation ».

À l'ensemble des bruits vocaux indiqués sur la partition correspond un effet mimétique précis, «décodifiable» seulement à travers la construction de chaînes associatives reproduites à l'infini (dynamiques/intensités sonores; signes d'agogique/modification de l'allure; variations dynamiques/gestes vocaux).

38 COHEN-LEVINAS, Danielle, La voix au-delà du chant, Paris, Vrin, 2006.p. 159. 
Figure 1 - Schéma "Système des signes et description gestuelle 39 », Lohengrin, azione invisibile

\begin{tabular}{|c|c|}
\hline $\begin{array}{l}\text { SYSTÈME DE SIGNES } \\
\text { (graphisme) }\end{array}$ & DESCRIPTION GESTUELLE40 \\
\hline & $\begin{array}{l}\text { Sussulto/sospiro } \\
\text { (sursaut/soupir) }\end{array}$ \\
\hline 2 & Sbadiglio (bâillement) \\
\hline & $\begin{array}{l}\text { Latrati/fiato/singhiozzi } \\
\text { (aboiements/souffle/sanglots) }\end{array}$ \\
\hline 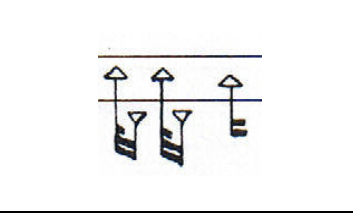 & $\begin{array}{l}\text { Saliva tra i denti/piccoli } \\
\text { schiocchi di lingua } \\
\text { (Salive parmi les dents/petits } \\
\text { claquements de langue) }\end{array}$ \\
\hline 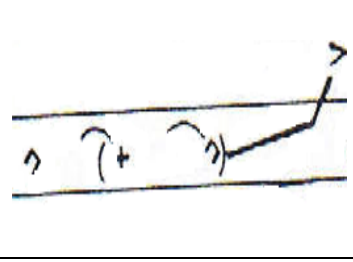 & $\begin{array}{l}\text { Fiato come onde }- \text { con } \\
\text { insensibili riprese di fiato } \\
\text { (souffle comme des ondes - } \\
\text { avec d'insensibles reprises de } \\
\text { respiration) }\end{array}$ \\
\hline$>$ & Colpi di gola (coups de gorge) \\
\hline & Colpi di tosse (coups de toux) \\
\hline$\frac{1}{k}$ & $\begin{array}{l}\text { Bolle di saliva/Gocce (boules de } \\
\text { salive/gouttes) }\end{array}$ \\
\hline$\vec{E}$ & $\begin{array}{l}\text { Battere di denti (battement de } \\
\text { dents) }\end{array}$ \\
\hline
\end{tabular}

39 Ce schéma fait référence aux signes repérés dans l'œuvre de Salvatore Sciarrino, Lohengrin, action invisible, Milan, Ricordi, 1984.

40 La description gestuelle du signe reprend l'indication en italien de Sciarrino dont nous donnons la traduction en français. 


\begin{tabular}{|l|l|}
\hline & Suono zero(son-zéro) \\
\hline & \\
\hline
\end{tabular}

Figure 2 - Schéma «Système d'indications d'expression », Lohengrin, azione invisibile, Perseo e Andromeda, Infinito nero.

\begin{tabular}{|c|c|c|}
\hline LOHENGRIN & $\begin{array}{c}\text { PERSEO E } \\
\text { ANDROMEDA }\end{array}$ & INFINITO NERO \\
\hline \multicolumn{3}{|c|}{ Indications Dynamiques } \\
\hline $\begin{array}{l}p, p p, m p, \text { poco, } f \text {, acc., lento e } \\
\text { accel., rall., } m p \text { (tesi), } p p p\end{array}$ & $\begin{array}{l}\text { ppp, } p, \text { mp, suono } \\
\text { zero, } m f \text {, } p \text {. sub., } \\
\text { pppp, pp, zero a mp, } \\
\text { zero a } p \text {, zero a } p p p \text {, } \\
\text { zero a } p p, \text { cresc., } \\
\text { poco cresc., accel., } f \text {, } \\
\text { zero a } f, \text { poco } m p, f f, \\
\text { fff }\end{array}$ & $\begin{array}{l}p p, p p p, p, p p p p \\
m p, f, m f\end{array}$ \\
\hline \multicolumn{3}{|c|}{ Indications d'expressions } \\
\hline $\begin{array}{l}\qquad \text { (32 voix) } \\
\text { Indications sonores } \\
\text { pp, più lontani (plus loin), mp, } \\
\text { più vicino (plus près), afono } \\
\text { (aphone), trepidante (anxieux), } \\
\text { sonoro (sonore), più sonoro, } \\
\text { diradandosi (en se dispersant), } \\
\text { leggero (léger). }\end{array}$ & $\begin{array}{l}\qquad \text { (18 voix) } \\
\qquad \underline{\text { I.S. }} \\
\text { eco (échos), leggero } \\
\text { (léger), leggerissimo } \\
\text { (très léger), sottovoce } \\
\text { (chuchoté), } \\
\text { glissando, urlato } \\
\text { (hurlé), nasale } \\
\text { (nasal), un poco } \\
\text { nasale (peu nasal). }\end{array}$ & $\begin{array}{l}\qquad \text { (6 voix) } \\
\qquad \text { I. S. } \\
\text { Subito } p \\
\text { (soudainement } p \text { ), } \\
\text { più } p \text { poss. (le plus } \\
\text { doucement } \\
\text { possible), sim. } \\
\text { (similairement) } \\
\text { afono (aphone), } \\
\text { sub. } f \text {. } \\
\text { (soudainement }\end{array}$ \\
\hline
\end{tabular}




\begin{tabular}{|c|c|c|}
\hline $\begin{array}{l}\text { Indications psychologiques } \\
\text { Materna (maternelle), concitata } \\
\text { (excitée), smarrita (égarée), } \\
\text { infantile (enfantine), assente } \\
\text { (absente), p, fra sé } \\
\text { (doucement, entre soi), con } \\
\text { infantile ebetudine, (avec une } \\
\text { hébétude enfantine), ride } \\
\text { argentina (rire argentin) } \\
\text { Indications physiologiques } \\
\text { con tubati (avec des } \\
\text { roucoulades), con sospiri (avec } \\
\text { des soupirs), con respiri (avec } \\
\text { des respirations), con } \\
\text { insensibili riprese di fiato (avec } \\
\text { d'insensibles reprises } \\
\text { d'haleine), si trasformano in } \\
\text { respiro (se transforment en } \\
\text { respirations), come soffocando } \\
\text { (comme pour s'étouffer), con } \\
\text { pressé et rythmique), più } \\
\text { risatina (avec un petit rire). } \\
\text { Indications rythmiques } \\
\text { pressante e ritmico (plus } \\
\text { rapide), ritmico e }\end{array}$ & $\begin{array}{l}\quad \underline{\text { I.P.41 }} \\
\text { dolcemente } \\
\text { (doucement), } \\
\text { fra sé (entre soi), } \\
\text { leggermente } \\
\text { (légèrement) } \\
\quad \text { I. R. } \\
\text { Libero (libre), } \\
\text { normale (normal), } \\
\text { troncato (coupé). }\end{array}$ & $\begin{array}{l}\text { fort). } \\
\qquad \underline{\text { I. R. }} \\
\text { molto rall. (très } \\
\text { ralenti). }\end{array}$ \\
\hline
\end{tabular}

41 Certaines indications que nous avons «cataloguées » comme psychologiques, peuvent faire également référence au son. Citons à titre d'exemple les expressions dolcemente (doucement) ou leggermente (légèrement) qui ont évidemment une double valence. C'est pourquoi nous soulignons que ce schéma est élaboré selon nos propres suggestions. 


\begin{tabular}{|c|c|c|}
\hline $\begin{array}{l}\text { uguale (rythmique et égal), } \\
\text { rapidissimo (très rapide), non } \\
\text { rapido (pas rapide), sempre } \\
\text { uguale (toujours pareil), p e } \\
\text { veloci le parole (doux, en } \\
\text { prononçant rapidement les } \\
\text { mots), stentando (forcé), } \\
\text { echeggiando (avec écho), } \\
\text { riprende (reprend), } \\
\text { s'interrompe (s'arrête), } \\
\text { s'interrompe bruscamente } \\
\text { (s'arrête brusquement). }\end{array}$ & & \\
\hline \multicolumn{3}{|c|}{ Gestes vocaux } \\
\hline (32 voix) & (6 voix) & (4 voix) \\
\hline $\begin{array}{l}\text { Voix signifiante } \\
\text { Detto rapido e nasale (parlé } \\
\text { rapide et nasal). }\end{array}$ & $\begin{array}{l}\quad \text { Voix bruit } \\
\text { Singhiozzando (avec } \\
\text { des sanglots). }\end{array}$ & $\begin{array}{l}\text { Voix signifiante } \\
\text { Cantato (chanté). }\end{array}$ \\
\hline Voix bruit & V. I. & V. I. \\
\hline $\begin{array}{l}\text { Singhiozzi (sanglots), } \\
\text { singhiozzi sempre più simili a } \\
\text { latrati lontani (sanglots de plus } \\
\text { en plus similaires à des } \\
\text { aboiements lointains), sussulto } \\
\text { (sursaut), rantolo (râle), tosse } \\
\text { (toux), risata (gros rire), pianto } \\
\text { (larmes), scoppio di pianto } \\
\text { infantile (éclat enfantin de } \\
\text { larmes), se pianto o riso è } \\
\text { incerto (larmes ou rire on ne }\end{array}$ & $\begin{array}{l}\text { Aria come sbadiglio } \\
\text { (soufflé tel un } \\
\text { bâillement), sbadiglio } \\
\text { (bâillement), solo } \\
\text { fiato (souffle seul), } \\
\text { quasi parlato } \\
\text { (presque parlé), } \\
\text { bocca chiusa (bouche } \\
\text { fermée). }\end{array}$ & $\begin{array}{l}\text { parlando nel } \\
\text { sospiro (parlé avec } \\
\text { un soupir), } \\
\text { inspirando (en } \\
\text { inspirant) } \\
\text { sospirando pppp } \\
\text { (en soupirant très } \\
\text { doucement). }\end{array}$ \\
\hline
\end{tabular}


sait pas), ma come un latrato (mais tel un aboiement), colpi di tosse (coups de toux), colpi di gola (coups de gorge), piccoli schiocchi di lingua (petits coups de langue), come un ticchettio (comme un crépitement).

\section{Voix intérieure}

Fiato (haleine), come onde (comme des vagues), sbadiglio (bâillement), fiato inspirando (souffle en inspirant), vento (vent), agitati sospiri (des soupirs animés), vento sulla schiuma (du vent sur l'écume).

\section{Voix animale}

verso di una tortora (roucoulement de tourterelle), pigolio (pépiement), latrati (aboiements), come latrati vicini (comme des aboiements proches), come latrati lontani (comme des aboiements lointains), batter di denti (claquer des dents), come un galoppo lontano (comme un galop lointain), tubando (roucoulant), a bocca chiusa come tubando (comme un 
roucoulement, bouche fermée).

\section{Voix signe}

Le labbra immobili (les lèvres serrées), come una bottiglia che si riempie (comme une bouteille qui se remplit), parlando (en parlant), schiudendo le labbra (ouvrant à peine les lèvres), saliva tra denti e labbra - bocca chiusa (salive parmi les dents et les lèvres - bouche fermée), bolle di saliva (boules de salive), come gocce echeggianti (comme des gouttes en écho), gocce sempre più copiose (gouttes de plus en plus nombreuses), gocciole (petites gouttes), violento (violent).

\section{Indication de timbre}

ce di Elsa infantile (voix d'Elsa enfant), voce di vecchio malato (voix de vieillard malade), voce di vecchia (voix de vieille dame), la voce di Lohengrin assume tono materno (la voix de L. prend un ton maternel) vecchio, rantolando (vieillard qui râle), altre voci (d'autres voix), bimbo, singhiozzando (avec des sanglots de bébé) 


\section{L'écoute: pouvoir de l'oreille et expérience écologique}

L'écoute est, d'après Daniel Teruggi, « une activité constante de notre perception ${ }^{42}$ » qui se fonde sur notre capacité à identifier les sources de sons, même sans les apercevoir visuellement. À l'ère de l'avènement de l'enregistrement, cette activité tant primordiale que naturelle s'est transformée en une véritable problématique, débordant du domaine strictement musical dans celui de la psychologie de l'écoute.

Avant de nous aventurer dans le monde sonore de l'infini possible et d'aborder la question de l'écoute au xxe siècle, une réflexion sur la signification du terme peut s'avérer très intéressante.

Le Dictionnaire de l'Académie française, en 1694, donne pour le verbe escouter la définition suivante: «ouïr avec attention, prester l'oreille pour ouïr ». Mais, étrangement, le substantif escoute ne désigne pas la simple et neutre action correspondant à ce verbe. II signifie : «lieu d'où l'on escoute sans estre veu». Le Larousse du XIXe siècle indique d'autres usages qui rapprochent l'écoute d'une activité de renseignement: «lieu fermé, dans un couvent, d'où l'on peut suivre l'office sans voir ni être vu ». L'écoute aurait été, dans son étymologie française, "une affaire de taupes ${ }^{43}$ », une pratique de l'auscultation s'approchant de l'espionnage dès la première écoute humaine ${ }^{44}$ : l'audition édénique ou adamique. Un siècle plus tard, l'Encyclopédie Einaudi ramène l'écoute à son historique proposant la distinction désormais connue de Roland Barthes. Ce dernier différencie, en effet, trois niveaux d'audition: l'écoute des indices (évaluation spatiotemporelle); l'écoute des signes (lecture des mêmes), et l'écoute de

42 TERUGGI, Daniel, «Tous les sons du monde. Le cadre de l'écoute acousmatique », Le renouveau de l'art total, Paris, L'Harmattan, 2004. Textes réunis et présentés par Danielle Cohen-Levinas, p. 131.

43 SZENDY, Peter, Sur écoute. Esthétique de l'espionnage, Paris, Les Éditions de minuit, 2007, p. 25.

44 Idem, p. 24. 
l'énonciation (tendue vers le «grain de la voix $45 »)$. Ce que l'écoute musicale, ainsi structurée, nous apprend sur l'écoute, c'est la solitude de toute évaluation qui n'a pas su s'ouvrir à une véritable interlocution. Si la première écoute est en fait très proche de l'âge de la peur ${ }^{46}$, la seconde écoute, elle, est un décryptage ; la troisième, qui «ne vise ou n'attend pas des signes déterminés classés", semble opérer une sorte de retour spiralé à l'origine. «En trois temps, en trois mouvements, l'ontologie de Barthes ressemble à une spirale de la peur 47 » dont la conception de Salvatore Sciarrino semblerait, d'une certaine façon, s'approcher.

Le compositeur ramène l'écoute à sa longue enfance; à «la pénombre des forêts et des cavernes obscures [...], [à] l'âge de la peur ${ }^{48} »$.

Une écoute archaïque ${ }^{49}$ qui n'est pas une menace de laquelle il faut se protéger, car cette mise en alerte est une représentation idéale de la puissance auditive. L'ampleur du développement des écoutes se mesurerait proprement à celle de la peur qui les habite. Cette conception, qui se ressent également de l'influence de Nietzsche, conçoit

45 Comme le note Barthes, évaluer le grain de l'autre, c'est aussi appeler l'autre à évaluer notre propre évaluation, c'est-à-dire notre propre corps, et à instaurer un échange où la plénitude des paroles s'équilibrera de la plénitude des silences. BARTHES, Roland, « Ascolta », Enciclopedia Einaudi, Turin, 1976.

46 Selon Nietzsche, il s'agit d'une forme d'écoute archaïque. NIETZSCHE, Friedrich, "Nuit et musique », Aurore. Réflexions sur les préjugés moraux, Paris, Gallimard, 1995, paragraphe 250.

47 SZENDY, Peter, op. cit., p. 49.

48 NIETZSCHE, Friedrich, «Nuit et musique », op. cit. L'oreille, organe de la peur, n'a pu se développer aussi amplement qu'elle l'a fait que dans la nuit ou la pénombre des forêts et des cavernes obscures, selon le mode de vie de l'âge de la peur, c'est-à-dire du plus long des âges humains qui ait jamais existé : à la lumière, l'oreille est moins nécessaire. D'où le caractère de la musique, art de la nuit et de la pénombre.

49 En fait, cette peur primordiale n'implique pas une sorte d'écoute passive à la façon d'Adorno car l'oreille ne doit pas se protéger des stimuli mais plutôt s'ouvrir à ceux-ci. Pour un approfondissement de la théorie d'Adorno, nous conseillons: ADORNO, Theodor, Wiesengrund, Introduction à la sociologie de la musique. Douze conférences théoriques, Genève, Contrechamps, 1994. Traduit par Vincent Barras et Carlo Russi. Dans cet essai, Adorno semble figer la vision et l'audition dans une opposition de nature physiologique: "L'oreille est passive. L'œil est recouvert par la paupière, on doit l'ouvrir; l'oreille est ouverte: plus que se tourner vers des stimuli d'une manière attentionnelle, elle doit s'en protéger [...]». 
l'écoute comme un phénomène physiologique et un «acte psychologique ${ }^{50}$ » qui reproduit et aggrave la dimension tragique de la communication.

Si l'écoute est, pour Sciarrino, une sorte de surveillance des possibles («la proie, la menace ou l'objet du désir qui passe sans prévenir $\left.{ }^{51} »\right)$, le moment le plus important est sans doute le déchiffrage du secret qu'elle cache. "Ce qui, enfoui dans la réalité, ne peut venir à la conscience humaine qu'à travers un code, qui sert à la fois à chiffrer cette réalité et à la déchiffrer 52 ».

Ce code peut être, dans l'idée de Sciarrino, tout à fait subjectif. Sa singularité consiste, en fait, dans l'introduction d'une écoute «moderne » développée dans un espace intersubjectif où «j'écoute » veut dire aussi «écoute-moi ${ }^{53}$ ». Une sorte d'audition globale, voire d'écoute totale, où on entend à la fois le moi et l'environnement écologique, l'autre. Cette intensification extrême de l'écoute dans sa forme hyperbolique représente une sorte de sur-écoute ${ }^{54}$, "qui s'ajoute par surcroît et qui vise d'emblée une pré-écoute ${ }^{55}$ ». La sur-écoute peut, en fait, se constituer comme une sorte de polyphonie de multiples lignes d'écoute qui se dédoublent, interfèrent et parfois se brouillent. Une superposition de voix, de bruits, de sons qui se mélangent dans le creux de notre oreille. Tendue vers la plus petite perception, cet organe évoque une sorte d'au-delà du noir impliquant l'ébauche d'un mouvement intérieur, d'un élan dynamique. Dans une telle situation, ce ne sont pas seulement les oreilles qui cherchent à voir, mais tout le corps. Cette extension du pouvoir des sens est le résultat d'une implication totale

50 BARTHES, Roland, «Ascolta », Enciclopedia Einaudi, Turin, Einaudi, 1976. Sous la direction de Ruggiero Romano et Corrado Vivanti.

51 BARTHES, Roland, "Écoute », repris dans /'Obvie et l'Obtus, Essais critiques III, Paris, Éditions du Seuil, 1982, p. 200.

52 Idem, p. 221.

53 Idem, p. 217.

54 SZENDY, Peter, Sur écoute. Esthétique de l'espionnage, Paris, Les Éditions de minuit, 2007, p. 26.

$55 / d e m$, p. 28. 
dans l'activité auditive tendue vers le moindre son au point que paraphrasant Bachelard 56 - les oreilles remplissent la fonction de voir aussi bien que d'entendre.

Cette forme d'attention perceptive hyperbolique est particulièrement accentuée dans l'obscurité totale. L'imagination est portée vers un au-delà du silence, de sorte que tout ce que l'on entend est rapporté à ce qu'on voit par imagination. II ne faut donc pas s'étonner de devenir oracle car « quand le sujet se donne tout entier à ses images, il aborde le réel avec une volonté aruspice 57 ». "À force d'entendre et de voir au-delà des sons et des choses, l'oreille révèle des transcendances, tout un au-delà de ce qu'on peut toucher » puisque comme l'affirme David Herber Lawrence - «l'oreille peut entendre plus profondément que les yeux ne peuvent voir58». Mieux on entend, moins on a besoin de voir.

$\mathrm{Si}$, effectivement, la nuit est le moment de la plus grande alerte, par syllogisme, l'oreille est bien le sens de la nuit.

Littérairement, la nuit est le moment négatif et de réflexion qui sert à la prise de conscience : on est en alerte et bien prédisposé à l'écoute puisque l'anxiété subtilise la perception [...] Allumer les sens, entrer en état d'alerte signifie avoir la perception globale du corps $[\ldots]^{59}$

\footnotetext{
56 Le commentaire de Bachelard du livre de Thomas Hardy, Le retour au pays natal, est à cet égard très significatif : « son attention était tendue à un degré tel que ses oreilles parurent presque remplir la fonction de voir aussi bien que d'entendre. On ne peut que constater cette extension du pouvoir des sens en un tel moment... il parvint à la suite d'un long entraînement à rendre son corps tellement sensible aux vibrations de l'air qu'il entendait par lui comme par des oreilles ». BACHELARD, Gaston, La terre et les rêveries du repos, Paris, Corti, 1948 , p. 156.

57 Idem, p. 87.

58 Cité in BACHELARD, Gaston, La terre et les rêveries du repos, p. 194. "The ears can hear deeper than eyes can see ».

59 GUERRASIO, Francesca, "Salvatore Sciarrino : entre esprit de la tradition et esprit contemporain», ResMusica, quotidien de la musique classique, 7 mai 2007. Entretien réalisé à Ravello en août 2004, consultable sur la page Internet www.resmusica.com.
} 
La conception de Salvatore Sciarrino (très proche de celle de Nietzsche) fait de cet espace temporel un espace intérieur: une nuit « d'outre noir », un « petit théâtre où s'affrontent deux déités modernes [...] : le pouvoir [de l'oreille] et le désir60 [d'une écoute globale] ». Dans ce lieu nocturne avec des points d'écoute inscrits et prescrits par l'architecture de l'œuvre, l'auditeur "sur-écoute »la totalité du tout : chaque bruit, chaque silence, chaque mot, la moindre respiration qui pourrait lui fournir un indice, lui-même, les protagonistes de la dramaturgie sonore, tous les personnages eux-mêmes à l'écoute, l'environnement circonstanciel. Cette «hypertension panacoustique 61 » qui voudrait percevoir le tout et n'en rien perdre, s'insère vers une «perspective tournante ${ }^{62}$ » de l'écoute. Tel le mouvement panoramique d'une caméra de surveillance, elle repère le moindre détail au point que l'œuvre devient un réseau incontrôlable où la menace est permanente. Dépassant les limites de l'écoute structurelle, cette écoute "panique », réduplication répétée dans l'écoute et de l'écoute elle-même, annonce la déconstruction d'une écoute totale comme d'un regard total. Ce qui resterait alors, comme l'écrit Peter Szendy, serait une écoute dissonante, non pas par rapport à la dissonance à proprement parler mais pour la dissonance qu'elle porterait en elle-même, pour sa duplicité irrésolue «sa fission non résorbée dans une fusion 63 ».

À l'époque où Nono, avec son Prometeo, crée une architecture pour l'écoute et pour l'écho (une sorte d'écho-tectonique ${ }^{64}$ ), Berio avec Un re in ascolto, mêle de façon calculée le lexique architectural et celui

60 SCIARRINO, Salvatore, Carte da suono, Rome-Palerme, Cidim-Novecento, 2002, p. 230.

61 SZENDY, Peter, Sur écoute, Paris, Les éditions de minuit, 2007, p. 147.

62 Idem, p. 55.

63 Idem, p. 148.

64 L'écho-tectonique est une architecture utilisée à des fins de surveillance auditive. Le père jésuite Athanasius Kircher dans sa Musurgia universalis, donne comme premier exemple d'écho-tectonique la grotte de Denys le Tyran. KIRCHER, Athanasius, Musurgia universalis, Rome, 1650, tome II, p. 291. Cité in SZENDY, Peter, Sur écoute, p. 32. 
de la description physiologique de l'oreille65; enfin, Sciarrino identifie, dans Lohengrin, le lieu d'écoute par excellence : «le merveilleux paysage de l'âme ». Intime et sans doute subjectif, il est le catalyseur de toutes les expériences d'écoute, de notre vécu, de la connaissance que nous avons du monde dans lequel nous vivons et de notre capacité à nous représenter le monde sans le voir. Un lieu qui nous informe sur ce que nous ne voyons pas et qui cherche en même temps à interpréter l'environnement circonstanciel.

Dans la reprise de I'œuvre, Lohengrin II, Sciarrino transfigure et élargit ce lieu d'écoute personnelle dans les jardins de la Villa Rufolo où les auditeurs se promènent en étant à l'écoute de tout ce qui est audible. D'une certaine façon, ils s'espionnent les uns les autres, formant ainsi un réseau "d'écouteurs » et de relais auditifs très complexe. Une écoute globale où chacun écoute la réalité avec « une oreille d'insecte et une de géant ${ }^{66}$ », déterminant ce que Sciarrino appelle une «expérience écologique». Cela ne peut avoir lieu que si l'on fait appel à une éducation de l'oreille qui conduise à « entendre correctement».

L'oreille toute seule est obtuse, n'a pas d'intelligence. L'esprit tout seul n'a pas de conscience. II faut être présents à nous-mêmes dans chaque action, en d'autres mots, adhérer à ce que nous faisons. [...] Les sons retrouvés près de l'horizon des sens, ceux du purgatoire infra-utérin, agrandis par l'ancien silence au travers la chute de la

\footnotetext{
65 «Le palais n'est que volutes et lobes, une grande oreille où anatomie et architecture échangent leurs noms et leurs fonctions: pavillons, trompes, tympans, colimaçons, labyrinthes, tu es tapi au fond, dans la zone la plus intérieure du palais-oreille, de ton oreille, le palais est l'oreille du roi ». CALVINO, Italo, Sous le soleil jaguar, Paris, Seuil, 1990, p. 62. Traduit de I'italien par Jean-Paul Manganaro.

66 SCIARRINO, Salvatore, Carte da suono, Rome-Palerme, Cidim-Novecento, 2002 , p. 26. Traduction personnelle de l'italien: "Ascoltando la realtà con un orecchio d'insetto e uno da gigante, cerco di restituirla in una musica di vento e pietra. Esperienza di ascolto queste, che, più di ogni altre, potrebbero definirsi ecologiche».
} 
mémoire, fluctuent et [nous, nous sommes] au milieu, et l'espace immuable émet des pulsations dans le noir67.

Cet appel à l'éducation est un motif récurrent, même chez Adorno, quoique la pensée de ce dernier aille plutôt à l'encontre d'une « dialectique auditive du détail68 », à savoir vers une écoute structurelle, que, nous le soulignons, Sciarrino refuse nettement. Selon Adorno il s'agit d'amener l'auditeur à saisir les compositions de manière structurelle, c'est-à-dire dans l'articulation de plusieurs moments sonores. Car c'est proprement dans cette articulation que « s'illumine une contexture de sens 69 ». L'écoute structurelle serait le déploiement nécessaire de la musique depuis le détail jusqu'à sa totalité. À partir de ce concept, le philosophe allemand formalise ensuite les instructions 70 pour l'écoute de la nouvelle musique. La compréhension de la musique est liée à la capacité de l'auditeur de percevoir des contextures (Zusammenhänge), de relier ce qui apparaît comme présent au passé et vice-versa : de percevoir la musique comme un tout plein de sens.

L'exigence [d'une écoute structurelle] s'annonce aujourd'hui de façon insistante et critique contre l'emprise du momentané, contre la mauvaise naïveté. Pré-artistique est l'écoute atomisée, qui se perd faiblement et passivement dans l'attrait de l'instant, dans

67 SCIARRINO, Salvatore, Carte da suono, Rome-Palerme, Cidim-Novecento, 2002, p. 53. Traduction personnelle de l'italien : "L'orecchio da solo è ottuso, non ha intelligenza. La mente da sola non ha coscienza. Dobbiamo essere presenti a noi stessi, in ogni azione. Con altre parole: aderire a ciò che facciamo. [...] i suoni ritrovati presso l'orizzonte dei sensi, quelli del purgatorio intrauterino, ingranditi per antico silenzio attraverso un crollo sommerso della memoria [...] fluttuano, e tu stai al centro, e uno spazio intatto ora pulsa nel buio».

68 SZENDY, Peter, Surécoute, Paris, Les éditions de minuit, 2007, p. 137.

69 ADORNO, Theodor, Wiesegrund, «Die gewürdigte Musik», Der getreue Korrepetitor. Lehrschriften zur musikalischen Praxis, Suhrkamp (Gesammelte Schriften, vol. 15), 1976, p. 184. Egalement cité in SZENDY, Peter, Surécoute, p. 137.

70 ADORNO, Theodor, Wiesegrund, "Anweisungen zum Hören neuer Musik», Suhrkamp (Gesammelte Schriften, vol. 15), 1976, p. 188-248. 
l'agréable sonorité isolée, dans la mélodie que l'on peut parcourir du regard et garder en mémoire ${ }^{71}$.

À cette capacité «structurelle » proposée par Adorno, Sciarrino substitue la faculté «mentale» de saisir, par l'oreille, toutes les images. La première écoute sert, en fait, à favoriser les migrations, à faire émerger les associations induites et non fournies par la mémoire. L'écoute est une mise à feu graduelle de la réalité à travers des modèles perceptifs transformés au fur et à mesure par l'expérience. En définitive, il s'agit pour notre perception de créer un contexte contenant les sons dans une logique combinatoire floue. L'idée d'un cadre d'écoute dans lequel notre imaginaire construit des images et des formes sonores à partir de ce que la musique lui suggère est très en ligne avec le concept d'écoute des sons sans référence visuelle: l'idée d'acousmatique. Dénuée de tout support visuel, la musique acousmatique est «suggérée et construite par l'auditeur 72 »; elle naît de sa capacité d'attribuer du sens aux sons, de concevoir une totalité signifiante faite seulement d'écoute.

[Cette] musique [est] faite seulement d'écoute, musique qui propose des mondes imaginaires à celui qui l'écoute à condition qu'il veuille rentrer dans sa diversité. Une musique qui utilise des sons réels ou des sons irréels, des sons captés dans la nature ou du monde instrumental, de sons de synthèse ou des sons aléatoires, tout est admis comme source ${ }^{73}$.

C'est à partir de là, à partir d'une écoute «propre », libérée de l'esclavage de la vue, que Sciarrino élabore son concept d'écoute

\footnotetext{
71 Repris par SZENDY, Peter, «Adorno, l'informateur aux écoutes », Expériences et fragments dans l'esthétique musicale d'Adorno, Paris, L'Harmattan, 2005, p. 51-60. Ouvrage collectif sous la direction de Jean-Paul Olive.

72 TERUGGI, Daniel, "Tous les sons du monde. Le cadre de l'écoute acousmatique », Le renouveau de l'art total, Paris, L'Harmattan, 2004, p. 136.

73 Idem, p. 137.
} 
écologique et d'écologie sonore. Réveillant dans l'auditeur la sensibilité à la différence infinie et l'attention à la «référence constitutrice » (Stoianova, 1988, p. 438), sa musique permet à nous tous de nous acheminer vers les infinis possibles.

\section{Conclusion}

L'atonalité et l'intégration du bruit au son - comme l'affirme Anne Roubet (2011, p. 620) - ont été les deux révolutions majeures du xxe siècle, surtout après la Seconde Guerre mondiale. La conjugaison de ces deux tendances, la naissance de la musique concrète et électronique et leur ambition de découvrir la musicalité potentielle des bruits, a suscité une aspiration nouvelle : découvrir dans le son musical la part de bruit qu'il contient. L'avant-garde des années 1960 semble en fait se partager entre la perpétuation d'un système formel appliqué aux douze sons du total chromatique (voire le sérialisme intégral) et la manipulation d'un monde sonore partiellement inédit à travers la musique électronique. Les traits marquants de la musique électronique "passés dans la musique instrumentale », tels la sculpture sonore, l'intériorité ou grain du son, les processus de transformation ou de modification sonores, sont à la base de nombreux langages à partir des années 1980 .

Une fois la dichotomie entre son et bruit supprimée, ce dernier, avec ses «prétendues impuretés» (Schaeffer, 1966, p. 670), devient partie intégrante du donné musical ou, mieux, de la matière sonore. Cet objet vivant qu'est le son évolue, se modifie, se transforme au travers de petites (presque imperceptibles) variations de timbre et des techniques d'entretien du son. Cela dans un temps dilaté, héritage de la musique spectrale, qui détermine un continuum sonore et une évolution continuelle.

La percussion et le percussif dans le son des instruments ajoutent des composantes de bruit blanc. L'emploi de registres extrêmes (hyper- 
grave ou hyper-aigu) et de divers modes de jeu (pizzicato, jeu sur le chevalet ou avec le bois de l'archet, pression rapide de l'archet, envolées d'harmoniques, sons écrasés aux cordes, avec l'ongle sous les touches du piano, Flatterzunge pour les vents, cluster de sons électroniques superposés aux sons instrumentaux, son multiphonique aux vents, micro-intervalles ${ }^{74}$ ) donnent lieu à des sons «sales » qui deviennent la marque de l'écriture de nombreux compositeurs, y compris de Sciarrino.

Lorsque celui-ci commence à écrire ses œuvres de maturité (au début des années 1980), il essaie de s'éloigner définitivement de l'esthétique postsérielle «fétichiste du symbole et de la note » (Levy, 2004 , p. 103) et du structuralisme 75 dominant. Ce dernier consiste dans l'analyse ou dans la création d'un objet selon les seules lois internes qui le structurent. Bien que les origines de la méthode structurelle remontent au début du siècle, on assiste à son apogée dans les décennies qui suivent la Deuxième Guerre mondiale. Principalement inscrit dans le domaine des sciences, il se développe rapidement même dans celui des arts où la structure devient non seulement une véritable « méthode créative » (Dosse, 1992, p. 256) basée sur l'abstraction de la conscience contextuelle du sujet, mais aussi le «levain de la modernité » (Dosse, 1992, p. 256). La primauté de la théorie résumée dans l'affirmation de Boulez «la musique n'est pas seulement destinée à exprimer, elle doit devenir le propre objet de sa réflexion » (Boulez, 1987, p. 33), implique la notion de « recherche musicale » liée à celle de progrès. Les expressions comme «valeur ajoutée », «multiplication », «module fixe », «structure de groupe » utilisées par de nombreux

\footnotetext{
74 La musique spectrale ajoute aux processus les progressions d'un spectre harmonique vers un spectre inharmonique, par décalage ou distorsion des fréquences, intégrant ou généralisant les micro-intervalles, les sons multiphoniques aux vents, riches en composants non harmoniques, les sons écrasés aux cordes, etc. Découvrir la pureté du son dans l'impureté est le but de certains compositeurs des années 1980, notamment de Grisey.

75 Le fondateur de l'école structurelle est Claude Lévi-Strauss. Dans l'introduction de son ouvrage Le Cru et le Cuit, il refuse et rejette radicalement les méthodes de la musique sérielle et de la musique concrète. LEVI-STRAUSS, Claude, Le cru et le cuit, Paris, Plon, 1963.
} 
compositeurs montrent la subordination de la musique aux techniques modernes, aux courants mathématiques et à la philosophie contemporaine. À l'inverse de l'artiste individualiste du XIXe siècle, les compositeurs du xxe siècle se définissent par rapport à la pensée scientifique de leur temps, appliquant à tous les aspects du métier musical des «normes de réflexion et d'élucidation» (Dufourt, 1986) propres à l'ère industrielle.

C'est proprement cet intellectualisme que Sciarrino refuse et rejette, basant sa "philosophie musicale » sur l'importance accordée à l'auditeur et à la notion de perception dans l'œuvre. Comme on le sait, même les musiciens de l'école spectrale ${ }^{76}$ et les musiciens acousmatiques prennent comme grammaire principale l'acoustique et/ou la perception. La musique spectrale, par exemple, se fonde sur l'utilisation de spectres sonores (harmoniques, inharmoniques ou fonctionnels) et sur la réalité acoustique pour rénover les procédés d'écriture et les notions élémentaires de théorie musicale. Les paramètres essentiels du son ne sont plus pensés comme des entités séparées, mais organisées en une méta-structure qui englobe leurs interrelations. Le spectre est un degré zéro de l'écriture qui recouvre les notions de hauteurs prise comme fréquences et mesurées en hertz ainsi que les notions de timbre ou d'harmonie, considérés dans la dépendance d'intensité des harmoniques et des partiels, des modes de jeu, par le biais des transitoires, du rythme et du tempo dans la dimension diachronique des métamorphoses du spectre sonore. Dans cette approche, l'échelle des fréquences est pensée comme un continuum; tout processus est pensé en termes fonctionnels (interpolation, dérivation, exponentiation, etc.) des techniques de filtrage, de

76 La musique spectrale - le terme est dû, semble-t-il, à Hugues Dufourt regroupe des compositeurs d'horizons variés. On attribue ses origines à Tristan Murail et à Gérard Grisey mais on peut citer des compositeurs comme George Benjamin, Jonathan Harvey, Philippe Hurel, Kaija Saariaho, Gilles Tremblay et Claude Vivier. Pour un approfondissement, nous conseillons JEDRZEJEWSKI, Franck, Dictionnaire des musiques microtonales, Paris, L'Harmattan, coll. «Univers musical », 2003, p. 224. 
désynchronisation, de distorsion et de tout ce qui permet de faire évoluer les spectres en jouant sur les relations d'équilibre interne du profil sonore. Quoique la notion de perception du phénomène sonore soit au centre de la musique spectrale, et bien que certaines partitions intègrent même des résultats d'expériences de psycho-acoustique, leur méthode est encore scientifique et surtout trop technologique pour séduire Sciarrino.

Sans doute la connaissance de Grisey et de ses expérimentations ont-elles eu une influence importante dans l'élaboration du langage de Sciarrino, mais il ne s'agit pas pour lui de la construction d'une grammaire formelle du sonore, ni de l'observation de résultats scientifiques sur le son et la perception du temps mais plutôt de la synthèse non structurelle entre tradition et contemporanéité.

Les sonagrammes et spectrogrammes qui mettent en évidence l'évolution continuelle du son de l'attaque à l'extinction, et le fait qu'à tout instant le rapport entre ses diverses composantes change, convoquent des spectres dynamiques, instables, mouvants [...] (Roubet, 2011 p.625).

Son exploitation sonore est davantage d'origine philosophique que scientifique. Cela afin de rendre l'œuvre accessible à tout auditeur. Si cette conception s'approche des préoccupations de Grisey mais aussi de Murail et de l'école répétitive, à savoir le souci d'élaborer un matériau sonore accessible à l'auditeur, cependant, contrairement à celle de ces derniers, sa musique n'est pas fondée sur des lois sonores issues de phénomènes acoustiques et psycho-acoustiques. Du moins pas de façon prioritaire. II nous semble que Sciarrino soit poussé, dans l'élaboration de ses œuvres, par la volonté de joindre les trois niveaux indiqués par Jean Molino (apud Nattiez, 1975): le niveau poḯtique (qui consisterait dans l'élaboration progressive d'un matériau à partir d'un autre précédent); le niveau neutre, et le niveau esthésique (qui consisterait dans la perception de l'œuvre par l'auditeur). Il exprime cela à travers la 
notion de processus, formalisée au début des années 1990 dans son essai sur les figures de la musique Le figure della musica da Beethoven a oggi, où il s'emploie pour recenser et comprendre la réalité perceptive d'autres compositeurs, afin de déduire formellement des processus ou des figures. Le processus qui désigne en général l'idée de développement est un mode de transformation graduelle allant d'un point à l'autre. Non pas une simple métamorphose d'une entité mais le passage d'un état à un autre qui se réalise dans et à travers le temps.

Le processus ainsi conçu a pour finalité de proposer une alternative au caractère ponctuel de la musique sérielle des années 1950. II s'agit d'un système ou d'une succession d'états (phases) distincts, qui possède une cohérence temporelle et une certaine forme, et qui est caractérisé par une situation perceptive. Pour qu'il y ait perception complète d'un processus, il faut définir ses extrémités afin de déduire le type de transformation. Les différents processus de mutation des sons ou de développement du silence semblent être à la base de la conception sonore de Sciarrino qui, comme nous l'avons dit, conçoit la musique comme un flux, un continuum sonore. Cette conception processuelle très proche de la notion de morphing, doit beaucoup à l'électroacoustique, aux musiques extra-européennes mais surtout, nous le répétons, à la musique spectrale et acousmatique. Une musique de transformations dynamiques semble pouvoir, en effet, dépasser la notion d'événement au profit du parcours.

Opposer structure et processus semble discutable puisqu'un processus repose sur une structure (voir la structure algorithmique d'un processus par exemple). En plus «la forme est comme l'enveloppe de la structure »(Bonnet, 2011, p. 540) ; un processus est «dialectique» (Bonnet, 2011, p. 540), une figure moderne du développement d'une situation qui ne s'épuise pas dans son énoncé liminaire mais est mobile par définition.

En fait, malgré l'envie de Sciarrino de dépassement d'une perception induite par la structure, face à ses figures nous sommes 
confrontés à des sortes de «structures » (mentales, idéales, pseudoscientifiques ou subjectives) déduites de la perception.

\section{Références}

ADORNO, Theodor. Ludwig, Wiesengrund, Théorie esthétique. Paris: Klincksieck, 1982, p. 255. Traduction de Marc Jimenez. Édition originale en allemand: Ästhetische Theorie. Francfort-sur-le-Main : Suhrkamp Verlag, 1970.

BARTHES, Roland. "Le grain de la voix», L'Obvie et l'obtus. Essais critiques III, Paris : Éditions du Seuil, 1982, p. 240. Réédition des Entretiens 1962-1980, éd. du Seuil, 1981.

BONNET, Antoine, Conditions et possibilités actuelles de la composition musicale. Thèse de doctorat, Université de Tours, 1991 cité in Eléments d'esthétique musicale, Paris: Actes Sud/Cité de la musique, 2011. p. 540. Sous la direction de Christian Accaoui.

BOSSEUR, Jean-Yves. Luciano Berio. In : KADAR, Georges ; BOSSEUR, Jean-Yves, etc. (org.), Musique de notre temps. Paris : Casterman, 1973.

BOSSSEUR, Jean-Yves. Vocabulaire de la musique contemporaine. Paris: Minerve, coll. « Musique Ouverte », 1996, deuxième édition, revue et augmentée. Première édition, Paris : Minerve, 1992.

BOSSIS, Bruno. Une représentation de l'écoute : Un re in ascolto di Luciano Berio. In: COHEN-LEVINAS, Danielle, Récit et représentation musicale. Paris: L'Harmattan, 2002, p. 256.

2005, p. 8 .

BOULEZ, Pierre. Penser la musique aujourd'hui. Paris : Gallimard, 1987.

DOSSE, François. Histoire du structuralisme. Paris : La Découverte, 1992, tome II.

DUFOURT, Hugues, «Pierre Boulez musicien de l'ère industrielle », Inharmonique $n^{\circ} \%$ : Le temps des mutations, Paris, IRCAM/Christian Bourgeois, 1986.

GINDT, Antoine (dir.). Le corps musical, Arles: Actes sud, 1990. [Ouvrage collectif sur Georges Aperghis].

LÉVY, Fabien. Tournant des années 70. De la perception induite par la structure aux processus déduits de la perception, Le temps de l'écoute, Paris: L'Harmattan, 2004, p. 103. Textes réunis et présentés par Danielle CohenLevinas. 
MOLINO, Jean, in NATTIEZ, Jean-Jacques, Fondements d'une sémiologie de la musique. Paris: éditions 10/18, 1975, p.50-62.

NONO, Luigi. Possibilité et nécessité d'un nouveau théâtre musical. Genève : Contrechamps n. 4, 1985, p. 55-67.

PETAZZI, Paolo. Appunti sul teatro musicale oggi in Italia. Sonus materiali per la musica contemporanea n. 163, Anno VII, déc. 1995, p. 10-30.

ROUBET, Anne, "Son et bruit », Eléments d'esthétique musicale, Paris: Actes Sud/Cité de la Musique, 2011. Ouvrage collectif sous la direction de Christian Accaoui.

SCHAEFFER, Pierre, Traité des objets musicaux: essai interdisciplinaires, Paris, Le Seuil, 1966, nouvelle édition.

STOIANOVA, Ivanka. L'exercice de l'espace et du silence, L'espace: Musique/Philosophie. Paris: L'Harmattan, 1998, p. 438. Textes réunis et présentés par Jean-Marc Chouvel et Makis Solomos.

VINAY, Gianfranco. "Vanités et dramaturgies négatives», Musique et dramaturgie, Esthétique de la représentation au xxe siècle, Paris: Publication de la Sorbonne, 2003, p. 813. Textes réunis sous la direction de Laurent Feneyrou. 